\title{
Comparison Between Fully and Partially Filled Dielectric Materials on the Waveguide of Circularly Polarised Radial Line Slot Array Antennas
}

\author{
Nishat Yasmin Koli ${ }^{1}$, Muhammad U. Afzal ${ }^{1}$, Karu P. Esselle ${ }^{2}$, and Md Zahidul Islam ${ }^{3}$ \\ ${ }^{1}$ School of Engineering, Macquarie University, Sydney, NSW, Australia \\ ${ }^{2}$ School of Electrical and Data Engineering, University of Technology Sydney, Sydney, NSW, Australia \\ ${ }^{3}$ Teleaus: Serveno Australia Pty Ltd, Sydney, NSW, Australia
}

mst-nishat-yasmin.koli@ students.mq.edu.au, muhammad.afzal@mq.edu.au, karu.esselle@uts.edu.au

\begin{abstract}
This paper presents an investigation on the waveguide of circularly polarised radial line slot array (RLSA) antennas to improve gain and radiation bandwidth. Two circularly polarised (CP) RLSA antennas were designed with two different waveguide configurations. In the first configuration the waveguide is fully filled with dielectric materials and in the second configuration the waveguide is partially filled with dielectric materials and rest of the waveguide is filled with air. Numerical results of these two CP-RLSA antennas with two different waveguide configurations are presented and compared. Significant improvements have been made in the 3$\mathrm{dB}$ directivity bandwidth and aperture efficiency of the antenna having waveguide partially filled with dielectric material. The 3-dB directivity bandwidth was measured $6.2 \%$ and aperture efficiency increased to $55.5 \%$. The CP-RLSA antenna has also achieved a peak directivity of $31.7 \mathrm{dBic}$ and a gain of 31.2 dBic as compared to the directivity 30.1 dBic and gain 29.5 dBic, respectively achieved with the CP-RLSA antenna having waveguide fully filled with dielectric material.

Index Terms-low-profile, radial line slot array, improved bandwidth, wireless communication, high gain, high aperture efficiency
\end{abstract}

\section{INTRODUCTION}

Recent years have witnessed a fast growing interest for satellite communication and wireless applications. In order to attract the competitive commercial market, highly efficient, low-cost, light weight antennas with wider radiation bandwidth are extremely desirable for satellite applications on mobile platform such as long distance trains, buses and aircrafts. Among the diversity of microwave and millimeter wave antennas, parabolic reflectors have been utilized for several years in satellite and terrestrial communication system. Parabolic reflectors provide high gain and good coverage but their bulky and large volume make them undesirable for mobile satellite application such as SATCOM, on-the-move connectivity. Also the non-planar configuration makes it difficult for transportation. The patch array antennas provide low-profile and planar configuration but the gain drops at higher frequency. The radial line slot array (RLSA) antenna was first introduced in Japan in 1980s as an alternative to parabolic dish used for direct broadcast satellite TV reception [1], [2]. Radial line slot arrays are slotted waveguide planar antennas with several advantages including high gain, high efficiency, low profile, and compact size. The RLSA antennas can be designed for linear, circular or elliptical polarization based on the slot layout in the top radiating surface. The antenna can be installed easily in roof-top on mobile vehicles and not subject to leaf or water build-up due to its planar surface. Many investigations were done to improve the performances of RLSA antennas [3]-[10]. In the conventional RLSAs, the waveguide was fully filled with dielectric materials [2]-[4]. The dielectric materials increase the weight and cost of the antenna. Also the thickness of the dielctric materials at higher frequency affects the antenna's radiation performance.

In this research, we have designed two circularly polarised radial line slot array (CP-RLSA) antennas with two different waveguide configuration. The motivation of this work is to design and compare the two different antennas' radiation performances. The design examples of these two antennas are explained in Section II. Predicted results such as near-field phase distributions and far-field patterns are given in Section III.

\section{CONFIGURATIONS OF CP-RLSA ANTENNAS}

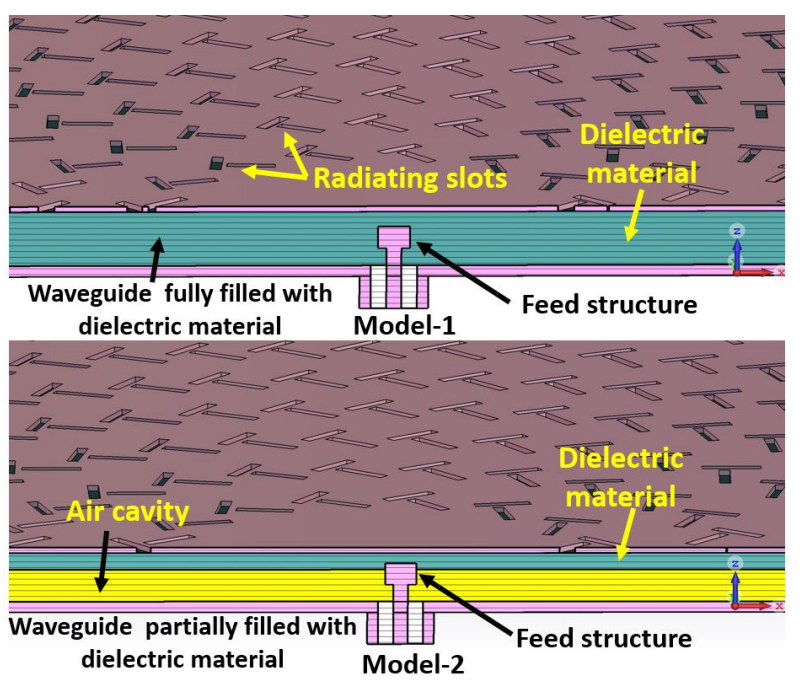

Fig. 1: Cross-section view of the two CP-RLSA antennas with two different waveguide configurations. 
The CP-RLSA antenna is made of two parallel conducting metal plates, forming a waveguide cavity inside these two plates. The upper plate is consisted of radiating slots and the bottom plate works as a ground plane. The waveguide is filled with dielectric materials of relative permittivity $\epsilon_{r}>1$ to create slow wave structure in the cavity with the guided wavelength, $\lambda_{g}$ to avoid grating lobes in the radiation patterns. The power is fed to the CP-RLSA antenna at the center of the ground plane with a $50 \Omega$ coaxial cable. The feed probe has a head-disk which resides inside the waveguide cavity. The radiating slots on the top plate radiate circular polarized electric fields.

Figure 1 shows the two CP-RLSA antennas with two different waveguide models operating at $20 \mathrm{GHz}$. In the first model (model-1) antenna, the waveguide is fully filled with dielectric materials. In the second model (model-2) antenna, the waveguide is partially filled with dielectric materials and rest of the waveguide is filled with air. The height of the waveguides were optimised to less than one-half of the guided wavelength $\left(\lambda_{g} / 2\right)$ to ensure the propagation of the transverse electromagnetic (TEM) wave. Table I shows the design parameters for the waveguide of the two models. In model-2, the average permittivity value was calculated from the dielectric material and air. All other design parameters were kept constant for these two antennas. Both models have an aperture size of $16 \lambda_{0}(240 \mathrm{~mm})$.

TABLE I: Design parameters of the two CP-RLSA antennas.

\begin{tabular}{|c|c|c|}
\hline Specification & Model-1 & Model-2 \\
\hline $\begin{array}{c}\text { Height of the } \\
\text { Waveguide }\end{array}$ & $4.5 \mathrm{~mm}$ & $4.5 \mathrm{~mm}$ \\
\hline Dielectric material & $\begin{array}{c}\text { Rogers } \\
\text { RT5880(lossy) }\end{array}$ & $\begin{array}{c}\text { Rogers } \\
\text { RT5880(lossy) }\end{array}$ \\
\hline $\begin{array}{c}\text { Thickness of the } \\
\text { dielectric material }\end{array}$ & $4.5 \mathrm{~mm}$ & $1.575 \mathrm{~mm}$ \\
\hline $\begin{array}{c}\text { Thickness of the air } \\
\text { cavity }\end{array}$ & 0 & $3 \mathrm{~mm}$ \\
\hline Permittivity & 2.2 & 1.41 \\
\hline Guided wavelength & $10.1 \mathrm{~mm}$ & $12.63 \mathrm{~mm}$ \\
\hline
\end{tabular}

\section{Simulation Results}

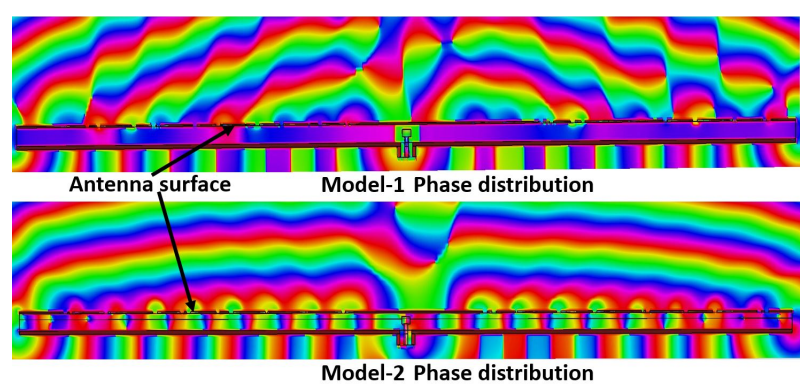

Fig. 2: Near-field phase distributions of the two antennas.

The two models were simulated in the transient solver of CST Microwave Studio from $18 \mathrm{GHz}$ to $20.5 \mathrm{GHz}$. Both near and far-field results were studied and compared. Both antennas have acceptable matching in the operating frequency range. Figure 2 shows the near-field phase distributions of the two CP-RLSA antennas at $20 \mathrm{GHz}$. The electric

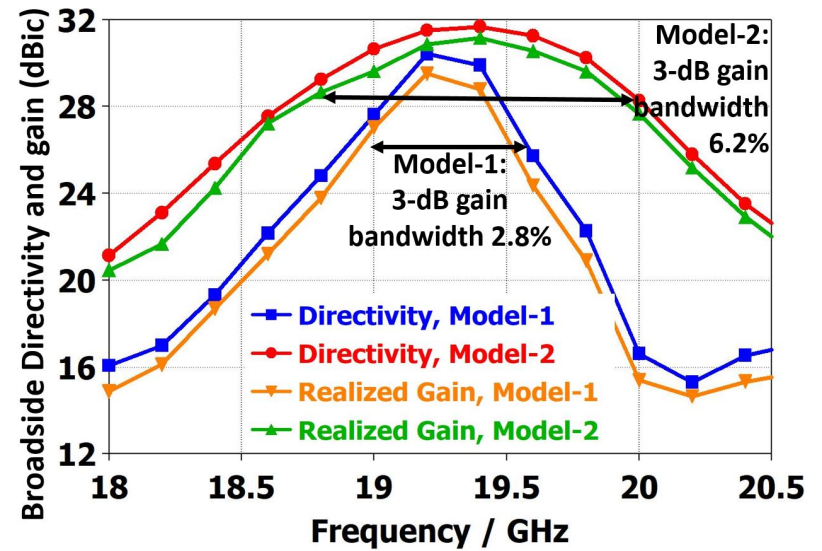

Fig. 3: Broadside far-field directivity and realized gain of the two CP-RLSA antennas.

near-field patterns $\left(E_{x}\right)$ were taken in the XZ plane at 20 $\mathrm{GHz}$, extending from the top plate to a distance of $2 \lambda_{0}(30$ $\mathrm{mm}$ above the top plate). As it can be seen from the figure, Model-1 has a non-uniform phase distribution throughout the aperture whereas Model-2 has shown more uniform phase distribution as compared to Model-1. The antennas have also successfully fulfilled the $3-\mathrm{dB}$ axial ratio condition of circular polarisation.

Figure 3 shows the broadside far-field directivity and realized gain of the two CP-RLSA antennas. Model-1 antenna has achieved a peak directivity of $30.4 \mathrm{dBic}$ with a relized gain of $29.5 \mathrm{dBic}$ at $19.2 \mathrm{GHz}$. Model-2 antenna has shown a peak broadside directivity $31.7 \mathrm{dBic}$ with a gain of 31.2 $\mathrm{dBi}$ at $19.4 \mathrm{GHz}$. Model-2 antenna has shown $1.3 \mathrm{~dB}$ more directivity and $1.7 \mathrm{~dB}$ more gain compared to Model-1 antenna. The 3-dB directivity bandwidth and 3-dB gain bandwidth of Model-1 antenna is only $2.8 \%$. Model-2 antenna has provided a $3-\mathrm{dB}$ directivity and $3-\mathrm{dB}$ gain bandwidth of $6.2 \%$ which is $3.4 \%$ more than the Model-1 antenna.

Figure 4 shows the 3D CST view of the radiation patterns of the model-1 and Model-2 antennas at $19.2 \mathrm{GHz}$ and 19.4 $\mathrm{GHz}$, respectively. The model-2 antenna has demonstrated a relatively good antenna efficiency. The aperture efficiency achieved with Model-1 antenna is 38\%, whereas Model-2 antenna has achieved an aperture efficiency of $55.5 \%$ which is $17.5 \%$ more than Model-1 antenna. Figure 5 shows the far-field radiation cut patterns of the two models at $19.2 \mathrm{GHz}$

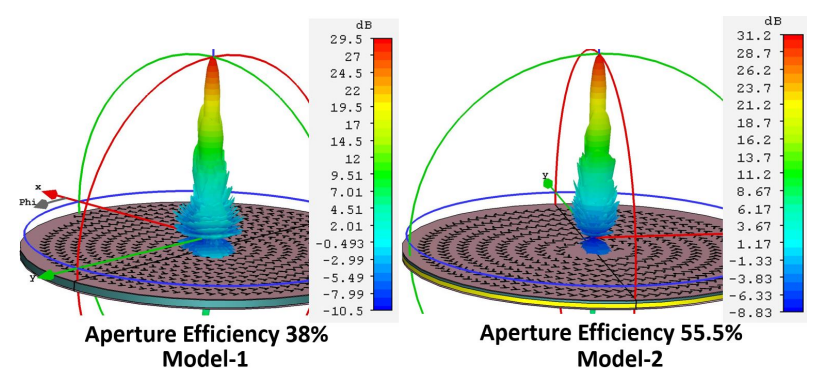

Fig. 4: 3D CST view of the radiation patterns of the two models. 


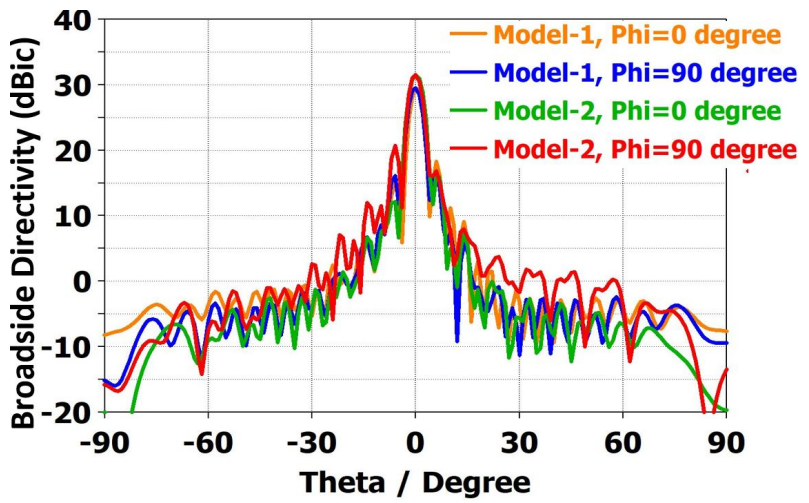

Fig. 5: Far-field radiation cut patterns of the two models at 19.2 GHz.

in both $\phi=0^{\circ}$ plane and $\phi=90^{\circ}$ plane. Model-1 antenna has provided a side lobe level (SLL) of $-11.2 \mathrm{~dB}$ in $\phi=0^{\circ}$ plane, whereas Model-2 has provided a side lobe level (SLL) of $-15.2 \mathrm{~dB}$ in $\phi=0^{\circ}$ plane. In $\phi=90^{\circ}$ plane, Model- 1 and Model-2 antennas have shown the SLLs of $-12.9 \mathrm{~dB}$ and -11 $\mathrm{dB}$, respectively.

\section{CONCLUSION}

Two CP-RLSA antennas were designed with two different waveguide configurations. The numerical results predict that the CP-RLSA antenna (Model-1) having the waveguide partially filled with dielectric material provides more uniform field distribution and good antenna performance compared to the antenna (Model-2) having the waveguide fully filled with dielectric material. The Model-2 CP-RLSA antenna has provided $1.3 \mathrm{~dB}$ more directivity and $1.7 \mathrm{~dB}$ more gain than the Model-1 antenna, which also increases the total aperture efficiency of Model-2 antenna (17.5\% more than Model-1 antenna). The 3-dB gain bandwidth of model-2 CP-RLSA antenna is $6.2 \%$ which is $3.4 \%$ more than Model-1 CP-RLSA antenna. The thickness of the dielectric material of the Model2 antenna is less compared to Model-1, which also reduces the total antenna weight.

\section{REFERENCES}

[1] M. Ando, K. Sakurai, N. Goto, K. Arimura, and Y. Ito, "A radial line slot antenna for $12 \mathrm{GHz}$ satellite TV reception," IEEE Transactions on Antennas and Propagation, vol. 33, no. 12, pp. 1347-1353, Dec 1985.

[2] M. Ando, K. Sakurai, and N. Goto, "Characteristics of a radial line slot antenna for $12 \mathrm{GHz}$ band satellite TV reception," IEEE Transactions on Antennas and Propagation, vol. 34, no. 10, pp. 1269-1272, 1986.

[3] P. W. Davis and M. E. Bialkowski, "Experimental investigations into a linearly polarized radial slot antenna for DBS TV in Australia," IEEE Transactions on Antennas and Propagation, vol. 45, no. 7, pp. 11231129, Jul 1997.

[4] P. W. Davis, "A linearly polarised radial line slot array antenna for direct broadcast satellite services," $\mathrm{PhD}$ dissertation, University of Queensland, Australia, 2000.

[5] P. W. Davis and M. E. Bialkowski, "Linearly polarized radial-line slotarray antennas with improved return-loss performance," IEEE Antennas and Propagation Magazine, vol. 41, no. 1, pp. 52-61, Feb 1999.

[6] M. N. Y. Koli, M. U. Afzal, K. Esselle, and M. Z. Islam, "A high gain radial line slot array antenna for satellite reception," in 2018 Australian Microwave Symposium (AMS), Feb 2018, pp. 65-66.

[7] M. Z. Islam, M. N. Y. Koli, and K. P. Esselle, "Design and improvement of pattern quality in circularly polarised slot array antenna for direct broadcast satellite applications," in 2017 2nd International Conference on Electrical Electronic Engineering (ICEEE), Dec 2017, pp. 1-4.
[8] J. M. F. Gonzalez, P. Padilla, G. Exposito-Dominguez, and M. SierraCastaner, "Lightweight portable planar slot array antenna for satellite communications in X-Band," IEEE Antennas and Wireless Propagation Letters, vol. 10, pp. 1409-1412, 2011.

[9] T. Nguyen, K. Sakurai, J. Hirokawa, M. Ando, O. Amano, S. Koreeda, T. Matsuzaki, and Y. Kamata, "A concise design of large mm-wave radial line slot antenna with honeycomb structures for space application," in 2014 XXXIth URSI General Assembly and Scientific Symposium (URSI GASS), Aug 2014, pp. 1-4.

[10] S. Peng, C. Yuan, T. Shu, and X. Zhao, "Linearly polarised radial line slot antenna for high-power microwave application," IET Microwaves, Antennas Propagation, vol. 11, no. 5, pp. 680-684, 2017. 